\title{
WEAK EQUALS STRONG FOR FIRST-ORDER LINEAR PSEUDODIFFERENTIAL BOUNDARY VALUE PROBLEMS
}

\author{
LEONARD SARASON
}

\begin{abstract}
Weak solutions are shown to be strong for a class of boundary value problems for first-order linear differential equations with zero order pseudodifferential matrix coefficients. The operators have the form $\chi A_{j}(x, D) \chi D_{j} \chi+\chi A_{0} \chi$, where $\chi$ is the characteristic function of a domain $G$. At the boundary, there are conditions on the coefficient $A_{n}$ of normal differentiation, typically implying that $\left(\chi A_{n}\right)^{-1}$ is a bounded operator both on $L_{2}(G)$ and on $H_{1}(G)$. The proof uses tangential mollifiers and is also applied in a more abstract setting.
\end{abstract}

Introduction. In studying 'weak equals strong' for boundary value problems for first-order linear systems of partial differential or pseudodifferential operators $([1],[3],[4],[5]$, and others), one of the principal tools has been the use of tangential mollifiers as introduced in [3]. Their success usually depends on the fact that the system of equations can be reduced to an equivalent one of the same type for which the coefficient $A_{n}$ of normal differentiation $D_{n}$ commutes with a standard tangential mollifier.

K. O. Friedrichs has suggested a different type of system of pseudodifferential operators, namely one in which the characteristic function $\chi$ of the domain stands between the differential operators $D_{j}$ and their pseudodifferential coefficients. Unless $A_{n}(x, D)$ has some special property however, such as satisfying the equation $\chi A_{n} \chi=\chi A_{n}$ (which would essentially reduce the system to a type considered by Agranovich [1]) or $\chi A_{n} \chi=A_{n} \chi$ (permitting one to multiply on the left by $A_{n}^{-1}$ if it exists and considered by Sarason and Tartakoff [5]), one reduces the coefficient of $D_{n}$ to one commuting with tangential mollifiers only by going outside the class of pseudodifferential operators. This procedure is carried out in $\S 1$ of the present paper. In $\S 2$, it is shown that strong convergence to zero of a sequence $\left[J_{n}, S\right]$ implies the same for sequences of the form $\left[J_{n}, f(S)\right]$, where $f$ belongs to a large class of analytic functions.

Received by the editors July 3, 1972.

AMS (MOS) subject classifications (1970). Primary 35S15; Secondary 41A35.

Key words and phrases. Tangential mollifiers.

(C) American Mathematical Society 1973 
1. Let $\Omega=x=\left(x^{\prime}, x_{n}\right): x_{n}<0$ be a halfspace in $R^{n}$, and denote the boundary of $\Omega$ by $\Gamma$. We shall work with spaces of vector-valued functions with values in $R^{N}$, in particular $L_{2}(\Omega), L_{2}(\Gamma), H^{1}(\Omega)$, and $H_{1,0}(\Omega)=H_{1,0}$, the latter defined by its norm

$$
\|u\|_{1,0}^{2}=\|u\|^{2}+\sum_{j \neq n}\left\|D_{j} u\right\|^{2},
$$

with the norms on the right-hand side above taken in $L_{2}(\Omega)$.

With $\chi$ the characteristic function of $\Omega$, set

$$
L=\sum \chi A_{j}(x, D) \chi D_{j} \chi+\chi A_{0}(x, D) \chi,
$$

where each $A_{j}$ is an $N$ by $N$ matrix of zero-order pseudodifferential operators of Kohn-Nirenberg type (we shall call such pseudodifferential operators $\psi \mathrm{DO}$ 's, and denote by $\psi D(s)$ the space of $\psi \mathrm{DO}$ 's of order $s$ ).

We assume that the leading symbol $A_{n}(x, \xi)$ of $A_{n}$ can be written as a product of symbols

$$
A_{n}(x, \xi)=\alpha(x, \xi) \beta(x, \xi) \mu(x),
$$

where $\alpha, \beta$ and $\mu$ have uniformly bounded inverses, are constant for large $x$, and where, moreover,

(i) for fixed $\left(x, \xi^{\prime}\right)$ with $\left|x_{n}\right|$ sufficiently small, $\alpha\left(x, \xi^{\prime}, \xi\right)$ is the boundary value of a function which is holomorphic in the upper half complex $\xi_{n}$-plane, and such that for each $\varepsilon>0$,

$$
\lim _{y \rightarrow+\infty} e^{-\varepsilon y} \sup _{\xi_{n} \text { real }}\left|\alpha\left(x, \xi^{\prime}, \xi_{n}+i \varepsilon y\right)\right|=0,
$$

(ii) $\beta\left(x^{\prime}, 0,0,1\right)=\beta\left(x^{\prime}, 0,0,-1\right)$, and

(iii) with some $k>0, \operatorname{Re}\left(\beta+\beta^{*}\right) \geqq k$ uniformly.

It follows from (i), (ii), and (iii), that $A_{n}(x, D)$ can be written as

$$
\alpha_{0} \beta_{0} \mu(x)+\gamma,
$$

where $\alpha_{0}$ and $\beta_{0} \in \psi D(0)$ and differ from $\alpha$ and $\beta$ by operators of order minus one, $\gamma$ has order minus one, and where

(iv) $\chi \alpha_{0}=\chi \alpha_{0} \chi$,

(v) $\chi\left(\beta+\beta^{*}\right) \chi \geqq k$ as an operator on $L_{2}(\Omega)$, and

(vi) $\chi \alpha_{0}$ and $\chi \beta_{0}$, considered as operators either from $L_{2}(\Omega)$ to itself or from $H^{1}(\Omega)$ to itself, are bounded and have bounded inverses, and $\chi \beta_{0}$ has a bounded inverse from $H_{1,0}$ to itself (see [5]).

We shall study first the equivalence of appropriately defined weak and strong solutions of the boundary value problem

$$
\begin{aligned}
L u=f & \text { in } \Omega, \\
u=\bar{u} & \text { on } \Gamma,
\end{aligned}
$$

where $f \in L_{2}(\Omega)$ and $\bar{u} \in L_{2}(\Gamma)$. 
Without loss, we assume that $\gamma=0$. This can be done because for functions $u \in H^{1}(\Omega), \chi \gamma \chi D_{n} \chi u$ is a bounded function of the pair $\left(u, u_{\Gamma}\right)$, where $u_{\Gamma}$ is the boundary value of $u$. In dealing with the more general boundary condition $P u=0$, we must make the additional assumption that $\chi \gamma \delta\left(x_{n}\right) \otimes(1-P)$ is a bounded map from $L_{2}(\Gamma)$ to $L_{2}(\Omega)$, as it will be, e.g. if $\gamma \in \psi D(-2)$.

In defining weak solutions of (1), we take advantage of the fact that Green's formula is known (see [2], [5]) in the case that $\alpha_{0}=I$. We shall say that the pair $(u, \bar{u}) \in L_{2}(\Omega) \times L_{2}(\Gamma)$ is a weak solution of (1) provided

$$
\begin{array}{ll}
\left(\chi \alpha_{0}\right)^{-1} L u=\left(\chi \alpha_{0}\right)^{-1} f & \text { in } \Omega, \\
u=\bar{u} & \text { on } \Gamma
\end{array}
$$

weakly in a sense to be defined below. This has the effect of taking the preclosed domain of the adjoint operator to be the set $\chi \alpha_{0}^{*} \chi H^{1}(\Omega)$.

Thus we can and do assume without loss that $\alpha=I$. We say that the pair $(u, \bar{u})$ is a weak solution of (1) if it satisfies Green's formula, namely

$$
(f, v)-\left(u, L^{*} v\right)=i\left(u, \mu^{*} \beta^{*} v\right)_{\Gamma}
$$

for all $v \in H^{1}(\Omega)$. We shall say that $(u, \bar{u})$ is a strong solution of (1) provided there is a sequence $u_{j} \in H^{1}(\Omega)$ such that

$$
\left\|u_{j}-u\right\|^{2}+\left\|L u_{j}-f\right\|^{2}+\left\|u_{j}-\bar{u}\right\|_{\Gamma}^{2} \rightarrow 0 \text { as } j \rightarrow \infty .
$$

THEOREM 1. $U=(u, \bar{u})$ is a weak solution of (1) if and only if $U$ is a strong solution.

Proof. That strong solutions are weak solutions is known. To prove that weak solutions are strong, we shall use tangential mollification.

Because $\beta^{*}$ satisfies the same conditions as $\beta$, we have that $\chi \beta \chi, \chi \beta^{*} \chi$, $\mu$ and $\mu^{*}$ are all bounded operators, both on $L_{2}(\Omega)$ and on $H^{1}(\Omega)$. In particular, it thus suffices to use in (2) test functions of the form $\left(\chi \beta^{*}\right)^{-1}\left(\mu^{*}\right)^{-1} v$ with $v \in H^{1}(\Omega)$; in this case (2) becomes

$$
\left(\mu^{-1}(\chi \beta)^{-1} f, v\right)-\left(u, L^{*}\left(\chi \beta^{*}\right)^{-1}\left(\mu^{*}\right)^{-1} v\right)=i(\bar{u}, v)_{\Gamma} .
$$

We wish to show that there exists a sequence $\left\{J_{m}\right\}$ of tangential mollifiers converging to $I$ and which has the property that the commutators

$$
\left[J_{m}, L^{*}\left(\chi \beta^{*}\right)^{-1}\left(\mu^{*}\right)^{-1}\right]
$$

tend strongly to zero in $L_{2}(\Omega)$. The existence of normal derivatives of the mollified functions $J_{m} u$ and convergence of their boundary values to $\bar{u}$ can be shown routinely, e.g., as in [4]. 
Remark 1. Essentially the same argument (see Lax and Phillips [3]) shows that weak equals strong for solutions of homogeneous boundary value problems with a boundary condition of the form $P u=0$, where $P$ is a bounded operator on $L_{2}(\Gamma)$ which commutes with translations. In this case the defining relation for weak solutions takes the form

$$
\left((\chi \beta \mu)^{-1} f, v\right)-\left(u, L^{*}\left(\chi \mu^{*} \beta^{*}\right)^{-1} v\right)=0
$$

for all $v$ such that $\left(1-P^{*}\right) v=0$ on $\Gamma$. For $u$ to be a strong solution in this set-up, the approximating functions $J_{m} u$ should satisfy $P J_{m} u=0$ on $\Gamma$, which they will provided $J_{m}$ commutes with $P$.

We take for $J_{m}$ the operator $\left(J_{m} u(x)\right)=m^{n-1} j\left(n\left(x^{\prime}-y^{\prime}\right)\right) u\left(y^{\prime}, x_{n}\right) d y^{\prime}$, where $j \in C_{0}^{\infty}\left(R^{n-1}\right)$ has average value one. The sequence of operators $\left\{J_{m}\right\}$ has the following properties:

(a) Each $J_{m}$ is a contraction and the sequence $\left\{J_{m}\right\}$ converges strongly to the identity on any of the spaces $L_{2}(\Omega), L_{2}(\Gamma), H^{1}(\Omega)$ and $H_{1,0}$.

(b) $\left[J_{m}, D_{j}\right]=\left[J_{m}, \chi\right]=0$.

(c) If $C$ is any zero-order $\psi \mathrm{DO}$ with a symbol compactly supported in $x$, then the sequences

$$
\left[J_{m}, C\right], D_{j}\left[J_{m}, C\right] \text { and }\left[J_{m}, C\right] D_{j} \quad(j \neq n)
$$

are uniformly bounded and converge to zero strongly as operators on $L_{2}(\Omega)$ and on $H_{1,0}$.

To prove (4), it suffices to show that with all zero-order $\psi$ DO's $C$ whose symbols have compact support in $x$,

$$
\left[J_{m}, \chi D_{j} C\left(\chi \mu^{*} \beta^{*}\right)^{-1}\right] \rightarrow 0 \quad \text { strongly in } L_{2}(\Omega), \quad j \neq n .
$$

To help prove (5), we shall need the following lemma.

LEMMA 1. As operators from $L_{2}(\Omega)$ to $H_{1,0},\left[J_{m}, \chi C\right]$ and $\left[J_{m}, \chi \beta^{*} \chi\right]$ are uniformly bounded in $m$ and tend to zero strongly as $m \rightarrow \infty$.

Lemma 1 is an immediate consequence of the listed properties of the sequence $\left\{J_{m}\right\}$.

To prove (5), we first write

$$
\begin{aligned}
& {\left[J_{m}, \chi D_{j}\left(\chi \mu^{*} \beta^{*}\right)^{-1}\right]} \\
& \quad=\chi D_{j}\left[J_{m}, \chi C\right]\left(\chi \mu^{*} \beta^{*}\right)^{-1}+\chi D_{j} C\left[J_{m},\left(\chi \beta^{*}\right)^{-1}\right] \mu^{*-1} \\
& \quad=\chi D_{j} C\left(\chi \beta^{*}\right)^{-1}\left[J_{m}, \mu^{*-1}\right] .
\end{aligned}
$$

The first and third terms on the right-hand side of (6) are easily seen to converge to zero strongly; because of Lemma 1 and the fact that $\chi C$ is a bounded operator on $H_{1,0}$, it suffices to show that the sequence $\left[J_{m},\left(\chi \beta^{*}\right)^{-1}\right]$ tends strongly to zero. 
The proof is by a homotopy argument. Since $\chi \beta^{*} \chi$ has a positive symmetric part, the operators

$$
S_{t}=(1-t) I+t \chi \beta^{*} \chi, \quad 0 \leqq t \leqq 1,
$$

have uniformly positive symmetric parts, and thus have inverses $T_{t}$ which are uniformly bounded. Further, we have

LEMMA 2. The operators $T_{t}: H_{1,0} \rightarrow H_{1,0}$ are uniformly bounded for $0 \leqq t \leqq 1$.

Proof. We claim that $\beta+\beta^{*}$ is a positive operator on $H_{1,0}$. To show this we use the equivalent norm $\|u\|_{\varepsilon>}$ defined as

If $u \in H_{1,0}$, then

$$
\|u\|_{\varepsilon>}=\left(\|u\|_{\Omega}^{2}+\varepsilon^{2} \sum_{j \neq n}\left\|D_{j} u\right\|^{2}\right)^{1 / 2} .
$$

$$
\begin{aligned}
(u, \beta u)_{\varepsilon}> & =(u, \beta u)-\varepsilon^{2} \sum_{j \neq n}\left(u, D_{j}^{2} \beta u\right) \\
& =(u, \beta u)+\varepsilon^{2} \sum_{j \neq n}\left(D_{j} u, \beta D_{j} u\right)+\varepsilon^{2} \sum_{j \neq n}\left(D_{j} u,\left[\beta, D_{j}\right] u\right) \\
& \geqq \frac{k}{2}\|u\|_{\varepsilon>}^{2}-p \varepsilon^{2} \sum_{j \neq n}\left\|D_{j} u\right\|\|u\|,
\end{aligned}
$$

where $p=\sup _{j \neq n}\left\|\left[\beta, D_{j}\right]\right\|$. Thus if $0<\varepsilon<(2 p n)^{-1}$, the form on the right is positive. Lemma 2 is proved.

The following lemma will conclude the proof of Theorem 1 .

Lemma 3. Let $\Lambda=\left(1-\sum_{j \neq n} D_{j}^{2}\right)^{1 / 2}$. There exists $\delta_{0}>0$ such that if $0<\delta<\delta_{0}$ and if $\Lambda\left[J_{m}, T_{t}\right] \rightarrow 0$ strongly on $L_{2}(\Omega)$, then $\Lambda\left[J_{m}, T_{t+\delta}\right] \rightarrow 0$ strongly as $m \rightarrow \infty$ with $0 \leqq t \leqq t+\varepsilon \leqq 1$.

Since $T_{0}=I$ commutes with $J_{m}$, the lemma ensures that $\Lambda\left[J_{m}, T_{1}\right]=$ $\Lambda\left[J_{m},\left(\chi \beta^{*}\right)^{-1}\right] \rightarrow 0$ strongly as $m \rightarrow \infty$.

Proof of Lemma 3. Set $\gamma=\chi\left(\beta^{*}-I\right) \chi$, and let

$$
M=\max \left(\|\gamma\|_{L_{2}(\Omega)},\|\gamma\|_{H_{1,0}}\right) \cdot \sup _{0 \leq t \leq 1}\left(\max \left\|T_{t}\right\|_{L_{2}(\Omega)},\left\|T_{t}\right\|_{H_{1,0}}\right) .
$$

If $0<\delta_{0}<(2 M)^{-1}$, we have, when $0<\delta<\delta_{0}$ and $t+\delta \leqq 1$,

$$
\begin{aligned}
\Lambda\left[J_{m}, T_{t+\delta}\right] & =\sum_{k=0}^{\infty} \Lambda\left[J_{m}, \delta^{k}\left(T_{t} \gamma\right)^{k} T_{t}\right] \\
& =\sum_{k=0}^{\infty} \Lambda\left[J_{m}, \delta^{k}\left(T_{t} \gamma\right)^{k}\right] T_{t}+\Lambda\left(1+\delta T_{t} \gamma\right)^{-1}\left[J_{m}, T_{t}\right] .
\end{aligned}
$$


The sequence $\Lambda\left(1+\delta T_{t} \gamma\right)^{-1}\left[J_{m}, T_{t}\right]$ converges strongly to zero because of Lemmas 1 and 2 . There remains to show the strong convergence to zero of $\sum\left[J_{m}, \delta^{k}\left(T_{t} \gamma\right)^{k}\right]$, which we expand as

$$
\delta\left(\sum_{k=1}^{K}+\sum_{k=K+1}^{\infty}\right) \Lambda\left(\sum_{r=s=1=k}\left(\delta T_{t} \gamma\right)^{r}\left[J_{m}, T_{t} \gamma\right]\left(\delta T_{t} \gamma\right)^{s}\right) .
$$

The sum $\sum_{K+1}^{\infty}$ can be estimated in norm by

$$
\delta \sum_{K+1}^{\infty} k(\delta M)^{k-1}\left\|\left[J_{m}, T_{t} \gamma\right]\right\|=\left.\delta\left\|\left[J_{m}, T_{t} \gamma\right]\right\| \frac{d}{d y} \frac{y^{K+1}}{1-y}\right|_{y=\delta M},
$$

which tends to zero as $K \rightarrow \infty$. Since the finite sum $\sum_{0}^{K}$ converges strongly to zero as $m \rightarrow \infty$, so also does the full sum.

REMARK 2. Because the operator $L$ differs from a sum of operators with support in coordinate patches by an operator of order minus infinity, Theorem 1 extends immediately to first-order linear operators of the same type on compact manifolds with boundary.

2. The argument given in $\S 1$ suggests a more general result on mollifiers which we now give, but in a more abstract setting.

Let $B$ and $B_{1}$ be Banach spaces with norms denoted by | | and by \| \| respectively such that $B_{1}$ is imbedded in $B$ and has the stronger topologyin particular, let $|u| \leqq\|u\|$ for all $u \in B_{1}$. Let $S$ be a bounded linear operator on $B$ mapping $B_{1}$ into itself and whose restriction to $B_{1}$, considered as an operator on $B_{1}$ and denoted by $S_{1}$, is also bounded. Denote the larger of the norms of $S$ and of $S_{1}$ by $\langle S\rangle$, and denote the union of the spectra of $S$ and of $S_{1}$ by $\sigma$.

Suppose that $\left\{J_{n}\right\}, n=1,2, \cdots$, is a sequence of linear operators from $B$ into $B_{1}$, each bounded in that sense, converging strongly to the identity as operators from either $B$ or $B_{1}$ to itself, and such that the commutators $\left[J_{n}, S\right]$, considered as operators from $B$ into $B_{1}$, are uniformly bounded:

$$
\left\|\left[J_{n}, S\right] u\right\| \leqq M|u|, \quad u \in B,
$$

and converge strongly to zero.

THEOREM 2. Let f be a function of one complex variable which is analytic on $\sigma$. Suppose that there exists a smooth simple closed Jordan curve $C$ such that $\sigma$ lies in the component of the plane interior to $C$, and such that $f$ is analytic on the interior component and on a neighborhood of $C$. Then the sequence $\left\{\left[J_{n}, f(S)\right]\right\}: B \rightarrow B_{1}$ tends strongly to zero.

Proof. Writing $f(S)$ as a contour integral

$$
f(S)=(2 \pi i)^{-1} \oint_{C}(z-S)^{-1} f(z) d z,
$$


we need information about the commutators $\left[J_{n},(z-S)^{-1}\right]$, and this information is furnished by the following lemma.

LEMMA 4. Suppose that zero lies in the unbounded component $U$ of the complement of $\sigma$. Then the commutators $\left[J_{n}, S^{-1}\right]$ tend strongly to zero as $n \rightarrow \infty$, and the quantity

$$
b=\sup _{n} \sup _{u \in B}\left\|\left[J_{n}, S^{-1}\right] u\right\| /|u|
$$

is finite and can be estimated from a knowledge only of $M,\langle S\rangle$, and the length $l$ and the minimum distance $d$ to $\sigma$ of some path $\gamma$ lying entirely in $U$ and which begins at 0 and ends at a point $z_{1}$ of modulus $2\langle S\rangle$. The estimate is improved by decreasing $l$ or by increasing $d$.

Proof of LÉma 4. The proof is an extension of the method of the proof of Lemma 3. We start by using the method of the proof of Lemma 3 to estimate $\left[J,\left(z_{1}-S\right)^{-1}\right]=z_{1}^{-1}\left[J,\left(1-z_{1}^{-1} S\right)^{-1}\right]$. Next we select a point $z_{2}$ on $\gamma$ at a distance $d / 2$ from $z_{1}$ (or set $z_{2}=0$ if $\left|z_{1}\right|<d / 2$ ). We want to estimate $\left[J,\left(z_{2}-S\right)^{-1}\right]$, which we write as

$$
\left[J,\left(1+\left(z_{2}-z_{1}\right)\left(z_{1}-S\right)^{-1}\right)^{-1}\left(z_{1}-S\right)^{-1}\right]
$$

and which equals

$$
\begin{aligned}
{\left[J,\left(1+\left(z_{2}-z_{1}\right)\left(z_{1}-S\right)^{-1}\right)^{-1}\right] } & \left(z_{1}-S\right)^{-1} \\
& +\left(1+\left(z_{2}-z_{1}\right)\left(z_{1}-S\right)^{-1}\right)^{-1}\left[J,\left(z_{1}-S\right)^{-1}\right] .
\end{aligned}
$$

The second term on the right-hand side above has in effect already been estimated, while to estimate the first, we again use the method of the proof of Lemma 3. Moving along $\gamma$ in this way and estimating at each step $\left\langle\left(z_{i}-S\right)^{-1}\right\rangle \leqq d^{-1}$, we arrive after a finite number of steps at an estimate for $\left[J, S^{-1}\right]$. That the estimate depends only on the listed quantities and in the prescribed way, is easily checked by the reader. Strong convergence of the sequence $\left\{\left[J_{n}, S^{-1}\right]\right\}$ to zero follows as in the proof of Lemma 3.

We can now conclude the proof of Theorem 2. Because of Lemma 4, the commutators $\left[J_{n},(z-S)^{-1}\right]$ are bounded independently of $n$ and of $z$ for $z$ on $C$. Further, one sees easily from the representation

$$
\begin{aligned}
& {\left[J,(z-S)^{-1}\right]-\left[J,\left(z^{\prime}-S\right)^{-1}\right]} \\
& =\left(z^{\prime}-z\right)\left(\left[J,(z-S)^{-1}\right]\left(z^{\prime}-S\right)^{-1}+(z-S)^{-1}\left[J,\left(z^{\prime}-S\right)^{-1}\right]\right)
\end{aligned}
$$

that these commutators are, for $z$ on $C$, uniformly Lipschitz continuous with Lipschitz constant independent of $n$.

It follows that the sequence of operators

$$
\left[J_{n}, f(S)\right]=(2 \pi i)^{-1} \int_{C}\left[J_{n},(z-S)^{-1}\right] f(z) d z
$$


can be approximated, uniformly in norm, by sequences of Riemann sums of the form

$$
(2 \pi i)^{-1} \sum_{j=1}^{N}\left[J_{n},\left(z_{j}-S\right)^{-1}\right] f\left(z_{j}\right)\left(z_{j}-z_{j-1}\right)
$$

with $z_{j}$ a fixed point on $C, j=1, \cdots, N$. Since the sequences $\left[J_{n},\left(z_{j}-S\right)^{-1}\right]$ converge strongly to zero, so also do the sequences of Riemann sums, and hence also their limits $\left[J_{n}, f(S)\right]$. Q.E.D.

\section{BIBLIOGRÄPHY}

1. M. S. Agranovič, On the theory of boundary value problems for symmetrizable firstorder systems, Mat. Sb. 73 (115) (1967), 161-197=Math. USSR Sb. 2 (1967), 143-170. MR 35 \#6998.

2. Louis Boutet de Monvel, Comportement d'un opérateur pseudo-différentiel sur une variété à bord. I, II, J. Analyse Math. 17 (1966), 241-304. MR 39 \#611; \#612.

3. P. D. Lax and R. S. Phillips, Local boundary conditions for dissipative symmetric linear differential operators, Comm. Pure Appl. Math.13 (1960), 427-455. MR 22 \#9718.

4. L. Sarason, On weak and strong solutions of boundary value problems, Comm. Pure Appl. Math. 15 (1962), 237-288. MR 27 \#460.

5. L. Sarason and D. S. Tartakoff, Boundary problems for non-elliptic first order systems of pseudo-differential operators, Comm. Pure Appl. Math. 25 (1972), 533-567.

6. K. O. Friedrichs, The identity of weak and strong extensions of differential operators, Trans. Amer. Math. Soc. 55 (1944), 132-151. MR 5, 188.

Department of Mathematics, University of Washington, Seattle, Washington 98195 